\title{
Low Dose Radiotherapy for COVID-19 Pneumonia: A New Perspective for an Old Horizon
}

\author{
Ahmad Ameri ${ }^{1,{ }^{*}}$, Nazanin Rahnama (i) ${ }^{1}$ and Farzad Taghizadeh-Hesary (iD ${ }^{2}$ \\ ${ }^{1}$ Department of Clinical Oncology, Imam Hossein Hospital, Shahid Beheshti University of Medical Sciences, Tehran, Iran \\ ${ }^{2}$ Department of Clinical Oncology, Shahid Beheshti University of Medical Sciences, Tehran, Iran \\ "Corresponding author: Department of Radiation-Oncology, Imam Hossein Hospital, Shahid Beheshti University of Medical Sciences, Tehran, Iran. Email: \\ ahmadameri1968@gmail.com
}

Received 2020 July 06; Accepted 2020 August 05.

Keywords: COVID-19, Pneumonia, Low-Dose Radiotherapy, Lung

\section{Dear Editor,}

SARS-CoV-2 pandemic is one of the biggest challenges of our time $(1,2)$, so that this incredible contagious and fatal virus has affected many aspects of human society. The key feature of COVID-19 infection, apart from its contagiousness, is the inflammatory cascade ignited by the immune system that causes the virus pathogenesis $(3,4)$. To suppress the uncontrolled inflammation, attention has been drawn to low dose radiotherapy (LDR), as a nonpharmacological treatment. It is a long time that LDR is used to treat benign conditions (5). LDR to the chest is currently under investigation in critically ill COVID-19 patients to alleviate the terminal condition $(6,7)$. As argued by Kirkby and Mackenzie (8), this field worth reconsidering in this crucial momentum. As previously demonstrated in animal and human experiments, low dose radiation can modulate the immune system to an anti-inflammatory condition (9). This phenomenon is not fully understood till now, but various mechanisms such as inhibition of certain pro-inflammatory cytokines (IL-6 in particular), reduction in chemotaxis of leukocytes, and shifting the balance to $\mathrm{m} 2$ macrophage lineage have been documented so far (10). The aforementioned mechanisms constitute fundamental parts of the COVID-19 mortality puzzle, and several studies have reported a "cytokine storm" (CS) caused by the SARS-CoV-2 infection $(11,12)$. To overcome the potentially fatal inflammation, low dose pulmonary irradiation, when administrated in due time and manner, can hypothetically reverse the process and prevent the inappropriate inflammatory response. The most significant side effect of radiation is the increased risk of secondary cancer. Increased rate of cancer has been reported in long term survivors who have received conventional-dose radiotherapy (13). Based on extrapolation of therapeutic dose data and experience from atomic accidents, researchers have attributed little excess relative risk of cancer to the radiation dose of less than 1 to $3 \mathrm{~Gy}$, especially in adults older than 60 years (14). Moreover, the latency period of radiationinduced cancer is at least 10 years.

Nevertheless, no treatment comes without adverse effects, and some risks have to be taken to achieve beneficial results. To address this matter, we have designed a pilot clinical trial at Shahid Beheshti University of Medical Sciences, Iran. According to the inclusion criteria, patients with moderate to severe confirmed COVID-19 pneumonia were recruited. The patients will receive low dose radiotherapy to the whole lungs in conjunction with the standard national protocol of COVID-19 management. The treatment will be planned using diagnostic chest CT scans. Variables of disease severity, such as baseline $\mathrm{PaO}_{2} / \mathrm{FiO}_{2}$ ratio, CBC, serum LDH, CRP, and IL-6 levels, are carefully documented, and radiation therapy is delivered in one fraction of $0.5 \mathrm{~Gy}$ to both lungs. In cases that clinical decision making in a multidisciplinary team is required, patients are allowed to receive an extra fraction of $0.5 \mathrm{~Gy}$ to a maximum of $1 \mathrm{~Gy}$ in 2 fractions at least 72 hours apart. We are expecting to witness the onset of radiation effects on clinical and serological variables within 48 hours; therefore patients will be closely monitored for 7 days after the radiotherapy session. A total of 5 patients will be enrolled, providing favorable results seen in the first set of patients, the team will decide on recruiting a larger number of participants for a phase II clinical trial. The complete study protocol as well as inclusion/exclusion criteria, could be found on

Copyright (c) 2020, Reports of Radiotherapy and Oncology. This is an open-access article distributed under the terms of the Creative Commons Attribution-NonCommercial 4.0 International License (http://creativecommons.org/licenses/by-nc/4.0/) which permits copy and redistribute the material just in noncommercial usages, provided the original work is properly cited. 
clinicaltrials.gov under the registry number NCT04390412. If proven effective, low dose RT can provide an alternative therapeutic option, which is readily available and can be offered to a large number of patients and, subsequently, reduce the burden of the pandemic on intensive care units, which is one of the biggest challenges of our time.

\section{Footnotes}

Authors' Contribution: AA designed the research. AA and NR performed the research. NR and FTH wrote the paper. All the authors reviewed and confirmed the final version of the paper.

Clinical Trial Registration Code: The clinical trial registration code was NCT04390412.

Conflict of Interests: The authors declare no conflict of interest.

Ethical Approval: This study was approved by the Ethical Committee of Imam Hossein Hospital (ethical code: IR.SBMU.RETECH.REC.1399.073).

Funding/Support: None declared by author.

Informed Consent: Prior to assigning the patients to radiotherapy, written informed consent was obtained.

\section{References}

1. World Health Organization. Global Surveillance for human infection with coronavirus disease (COVID-19). 2020. Available from: https://www.who.int/publications-detail/global-surveillance-forhuman-infection-with-novel-coronavirus-(2019-ncov.

2. Shankar A, Saini D, Roy S, Mosavi Jarrahi A, Chakraborty A, Bharti SJ, et al. Cancer care delivery challenges amidst coronavirus disease-19 (COVID-19) outbreak: specific precautions for cancer patients and cancer care providers to prevent spread. Asian Pacific Journal of CancerPrevention.2020;21(3):569-73. doi:10.31557|APJCP.2020.21.3.569. [PubMed: 32212779].
3. Cheng H, Jian S, Liu D, Ng T, Huang W, Lin H. Contact tracing assessment of COVID-19 transmission dynamics in Taiwan and risk at different exposure periods before and after symptom onset. JAMA internal medicine. 2020. doi: 10.1001/jamainternmed.2020.2020. [PubMed: 32356867].

4. Qin C, Zhou L, Hu Z, Zhang S, Yang S, Tao Y, et al. Dysregulation of immune response in patients with COVID-19 in Wuhan, China. Clinical In fectious Diseases. 2020. doi: 10.1093/cid/ciaa248. [PubMed: 32161940].

5. Lara PC, Burgos J, Macias D. Low dose lung radiotherapy for COVID19 pneumonia. The rationale for a cost-effective anti-inflammatory treatment. Clinical and Translational Radiation Oncology. 2020. doi: 10.1016/j.ctro.2020.04.006. [PubMed: 32373721].

6. Kefayat A, Ghahremani F. Low dose radiation therapy for COVID-19 pneumonia: a double-edged sword. Radiotherapy and Oncology. 2020. doi:10.1016/j.radonc.2020.04.026. [PubMed:32342874].

7. Salomaa S, Bouffler SD, Atkinson MJ, Cardis E, Hamada N. Is there any supportive evidence for low dose radiotherapy for COVID-19 pneumonia? International Journal of Radiation Biology. 2020;(just-accepted):119. doi:10.1080/09553002.2020.1786609.

8. Kirkby C, Mackenzie M. Is low dose radiation therapy a potential treatment for COVID-19 pneumonia? Radiotherapy and Oncology. 2020. doi: 10.1016/j.radonc.2020.04.004. [PubMed: 32342871].

9. Frey B, Hehlgans S, Rödel F, Gaipl US. Modulation of inflammation by low and high doses of ionizing radiation: implications for benign and malign diseases. Cancer letters. 2015;368(2):230-7. doi: 10.1016/j.canlet.2015.04.010. [PubMed: 25888451].

10. Arenas M, Sabater S, Hernández V, Rovirosa A, Lara PC, Biete A, et al. Anti-inflammatory effects of low-dose radiotherapy. Strahlentherapie und Onkologie. 2012;188(11):975-81. doi: 10.1007/s00066-012-0170-8. [PubMed: 22907572].

11. Mehta P, McAuley DF, Brown M, Sanchez E, Tattersall RS, Manson JJ, et al. COVID-19: consider cytokine storm syndromes and immunosuppression. Lancet (London, England). 2020;395(10229):1033. doi: 10.1016/S0140-6736(20)30628-0.

12. Taghizadeh-Hesary F, Akbari H. The powerful immune system against powerful COVID-19: A hypothesis. Medical Hypotheses. 2020:109762. doi: 10.1016/j.mehy.2020.109762. [PubMed: 32388390].

13. Brenner DJ, Sachs RK. Estimating radiation-induced cancer risks at very low doses: rationale for using a linear no-threshold approach. Radiation and environmental biophysics. 2006;44(4):253-6. doi: 10.1007/s00411-006-0029-4. [PubMed: 16470411].

14. Jansen JTM, Broerse JJ, Zoetelief J, Klein C, Seegenschmiedt HM. Estimation of the carcinogenic risk of radiotherapy of benign diseases from shoulder to heel. Radiotherapy and oncology. 2005;76(3):270-7. doi:10.1016/j.radonc.2005.06.034. [PubMed: 16157402]. 